\title{
Determination of Caveolin-1 in Renal Caveolar and Non-Caveolar Fractions in Experimental Type 1 Diabetes
}

\author{
H. DEMOVÁ, R. KOMERS \\ Diabetes Center, Institute for Clinical and Experimental Medicine, Prague, Czech Republic
}

Received September 6, 2007

Accepted May 22, 2008

On-line July 25, 2008

\section{Summary}

Caveolin-1 (CAV-1) is the main structural component of caveolae, acting as a modulator of signal transduction. CAV-1 might be involved in the pathophysiology of microvascular complications in Type 1 diabetes (DM). We sought to determine whether fractionation on sucrose gradient (SF), a method routinely utilized for isolation of caveolar fractions in homogenous cell lines, is applicable for CAV-1-related studies in tissues with multiple cell types, such as the normal rat kidney cortex (C). Using this method, we also determined whether streptozotocininduced DM in rats (4-week duration) leads to changes in renal subcellular targeting of CAV-1, and evaluated the effects of tight metabolic control (insulin, $12 \mathrm{IU} /$ day) and angiotensin receptor blocker, losartan (4 weeks, $20 \mathrm{mg} / \mathrm{kg} /$ day). Immunoblotting of individual fractions obtained from $\mathrm{C}$ revealed CAV-1 expression in fractions 4-6 that corresponded to light scattering band that typically forms after separating cellular fractions on SF. These fractions were considered to be caveolar fractions. In C, CAV-1 was also detectable in fractions 8-10. These and all other fractions except caveolar fractions were considered to be noncaveolar fractions. A ratio of caveolar/non-caveolar expression of CAV-1 (CNCR) was computed for each renal cortex allowing comparisons of CAV-1 subcellular distribution in C and DM rats, and effects of treatments. Using this approach, DM was characterized by marked increases in CNCR as compared to C $(5.54 \pm 1.56$ vs. $2.65 \pm 1.33, \mathrm{p}<0.05)$ that were reduced by treatment with insulin $(0.78 \pm 0.24, \mathrm{p}<0.01 \mathrm{vs}$. DM) or losartan $(0.84 \pm 0.06, p<0.01$ vs. DM). In summary, analysis of CAV- 1 following the SF of renal cortex detected similar distribution of the protein as in homogenous cell lines, DM-induced changes in CAV-1 targeting, and the effects of pharmacological treatments. This suggests applicability of SF in studies focusing on CAV-1 targeting in organs with various cell lines in vivo.

\author{
Key words \\ Caveolin-1 • Type 1 diabetes mellitus • Kidney disease • Sucrose \\ fractionation • Insulin • Losartan
}

\section{Corresponding author}

R. Komers, Diabetes Center, Institute for Clinical and Experimental Medicine, Vídeňská 1958, Prague 4, Czech Republic. Fax: 4202-26136-3183. E-mail: komersr@ohsu.edu

\section{Introduction}

Caveolae are plasma membrane invaginations in a variety of cell types (Anderson 1998). Caveolae act as signaling platforms, serve as concentrating points for numerous signaling molecules, and regulate flux through many distinct signaling cascades (Anderson 1998, Frank et al. 2003). Caveolin-1 (CAV-1) is the main structural protein component of caveolae (Anderson 1998, Frank et al. 2003). Localization of signaling molecules to caveolae involves direct interactions with the scaffolding domain of CAV-1 (Engelman et al. 1998). Protein-protein interactions of CAV-1 with these molecules have major impact on their catalytic functions and intracellular targeting. Consequently, CAV-1 appears to be an important modulator of a wide spectrum of signaling pathways.

Metabolic and humoral changes in Type 1 diabetes mellitus (DM) lead to hemodynamic, biochemical and structural changes in the kidney (Cooper 1998). Factors characteristic to diabetic metabolic milieu, such as hyperglycemia, glycosylation products, lipids and fatty acids contribute to changes in signal transduction pathways resulting in a wide spectrum of intracellular 
functional, biochemical changes, and induction of genes within the affected cells. In concert, these consequences of altered intracellular signaling in DM trigger and perpetuate functional and morphological alterations in the diabetic kidney ultimately resulting in renal failure. As a major modulator of signal transduction, CAV-1 could play important roles in DM-induced alterations in intracellular signaling and enzymatic function, and consequently have an impact on the development of microvascular complications. However, CAV-1 has not been so far extensively studied in this context. In the present studies we pursued two aims. First, we sought to determine whether sucrose fractionation, a method routinely utilized for isolation of caveolar fractions in homogenous cell lines, is applicable for CAV-1 related studies in parenchymatuos organs with a variety of cell types, such as the kidney. Second, applying this method, we sought to determine whether experimental DM1 leads to changes in renal subcellular targeting of CAV-1, and elucidate potential effects of insulin and angiotensin $\mathrm{AT}_{1}$ receptor blocker (AT1R), losartan, as standard treatments for DM1 and diabetic nephropathy.

\section{Methods}

\section{Diabetic rat model}

Studies were conducted in adult male Wistar rats (Anlab, Prague, Czech Republic) with initial body weight about $250 \mathrm{~g}$. The rats were made diabetic by intraperitoneal injection of streptozotocin (Sigma, St. Louis, MO), $65 \mathrm{mg} / \mathrm{kg}$ body weight. Three days later, induction of diabetes was confirmed by measurements of tail blood glucose (BG) level using a reflectance meter (One Touch II, Lifescan, Milpetas, CA). The animals were housed with a light-dark cycle of $12 \mathrm{~h}$ each, and with free access to food (standard chow) and water.

\section{Study design}

Diabetic rats were randomized to receive no insulin treatment (DM-0, n=4), 12 IU of insulin/day (DM12, Insulatard, Novo Nordisk, Copenhagen, Denmark, $\mathrm{n}=4$ ), to achieve tight metabolic control, or losartan (DM0+LOS, $20 \mathrm{mg} / \mathrm{kg} /$ day in drinking water). Age-matched non-diabetic Wistar rats served as controls $(n=4)$. Body weight, blood glucose and systolic blood pressure (SBP, tail plethysmography) were measured at week 4 after the induction of diabetes. Two to three days following these measurements, the rats were sacrificed by cervical dislocation and the kidneys were exposed via midabdominal incision, removed, divided into cortical and medullary portions, and snap frozen in liquid nitrogen for further analyses. All experiments were carried out with the approval of, and in accordance with the regulations of, the Institutional Animal Care and Use Committee of the Institute for Clinical and Experimental Medicine.

\section{Tissue fractionation on sucrose gradient}

Renal cortical samples (100 mg) were homogenized in $1.5 \mathrm{ml} 0.5 \mathrm{M} \mathrm{Na}_{2} \mathrm{CO}_{3}$ buffer ( $\mathrm{pH} 11$ ) containing $1 \mathrm{mM}$ EDTA, $1 \mathrm{mM} \mathrm{Na} \mathrm{VO}_{4}, 1 \mathrm{mM}$ DTT, $1 \mathrm{mM}$ PMSF, $1 \mathrm{mM} \mathrm{NaF}, 10 \mathrm{nM}$ okadaic acid, $10 \mu \mathrm{g} / \mathrm{ml}$ aprotinin, and $10 \mu \mathrm{g} / \mathrm{ml}$ leupeptin. The homogenate ( $1 \mathrm{ml}$ ) was placed in a $17-\mathrm{ml}$ ultracentrifuge tube and was adjusted to $45 \%$ sucrose by adding $1 \mathrm{ml}$ of $90 \%$ sucrose in MBS (25 mM MES, pH 6,5, $150 \mathrm{mM} \mathrm{NaCl).} \mathrm{Sucrose}$ gradient was prepared by adding $4 \mathrm{ml}$ of $35 \%$ sucrose in MBS and $4 \mathrm{ml}$ of $5 \%$ sucrose in MBS on top of it. Tubes were centrifuged at $39,000 \mathrm{rpm}$ at $4{ }^{\circ} \mathrm{C}$ for $24 \mathrm{~h}$. Lightscattering bands corresponding to caveolar fractions (Lisanti et al. 1994, Song et al. 1996) were clearly visible after centrifugation. One milliliter samples corresponding to fractions 1-10 were collected from the top to the bottom of each tube (Lisanti et al. 1994, Song et al. 1996) and stored in $-70{ }^{\circ} \mathrm{C}$ for further analyses. Protein concentration in each fraction was determined using a method by Lowry et al. (1951).

\section{Western blot analysis}

An equal volume of each fraction was mixed with loading buffer (0.5 M TRIS/HCl, $10 \%$ SDS, glycerol, 0.1 $\%$ bromphenol blue) and boiled for $5 \mathrm{~min}$, followed by Western blot analysis as previously described (Komers et al. 2006). In brief, denatured proteins were separated through an SDS-polyacrylamide gel and transferred to PVDF membranes (Bio-Rad Laboratories, Hercules, CA). Membranes were washed and then blocked overnight with TRIS buffered saline, plus $0.05 \%$ Tween-20 (TBS-T) containing $5 \%$ nonfat dry milk. Following blocking, membranes were again washed, and incubated overnight with rabbit polyclonal anti-Cav1 (Santa Cruz Biotechnology, Santa Cruz, CA), diluted 1:800 in TBS-T. Immunodetection was accomplished by incubating membranes with a goat anti-rabbit-IgG secondary antibody conjugated with horseradish peroxidase (HRP) for $60 \mathrm{~min}$ (1:100,000 Pierce) in TBS-T containing $5 \%$ nonfat dry milk. Visualization was performed with enhanced chemiluminescence (ECL) Western-blotting kit (Supersignal West Dura, Pierce) according to the 
Table 1. General characteristics control and diabetic rats.

\begin{tabular}{lllllll}
\hline & $\mathbf{n}$ & $\begin{array}{l}\text { BWT } \\
{[\mathbf{g}]}\end{array}$ & $\begin{array}{l}\mathbf{R K W} \\
\mathbf{[ g ]}\end{array}$ & $\begin{array}{l}\text { RKW/ } \\
\mathbf{1 0 0 g} \mathbf{B W T}\end{array}$ & $\begin{array}{l}\text { BG } \\
{[\mathbf{m m o l} / \mathbf{l}]}\end{array}$ & $\begin{array}{l}\text { SBP } \\
{[\mathbf{m m} \mathbf{H g}]}\end{array}$ \\
\hline Control & 6 & $356 \pm 7$ & $1.10 \pm 0.04$ & $0.31 \pm 0.02$ & $5.4 \pm 0.1$ & $121 \pm 5$ \\
$D M-0$ & 6 & $268 \pm 19^{\mathrm{be}}$ & $1.39 \pm 0.08^{\mathrm{b}}$ & $0.52 \pm 0.02^{\mathrm{be}}$ & $22.0 \pm 1.0^{\mathrm{be}}$ & $149 \pm 12^{\mathrm{a}}$ \\
$D M-12$ & 6 & $310 \pm 7^{\mathrm{b}}$ & $1.07 \pm 0.03^{\mathrm{d}}$ & $0.35 \pm 0.02^{\mathrm{d}}$ & $7.2 \pm 2.0^{\mathrm{b}}$ & $143 \pm 8^{\mathrm{a}}$ \\
$D M-0+L O S$ & 6 & $250 \pm 13^{\mathrm{be}}$ & $1.15 \pm 0.10^{\mathrm{c}}$ & $0.46 \pm 0.02^{\mathrm{ae}}$ & $19.3 \pm 1.3^{\mathrm{be}}$ & $118 \pm 5^{\mathrm{ce}}$ \\
\hline
\end{tabular}

BWT, body weight; RKW, right kidney weight; BG, blood glucose; SBP, systolic blood pressure.

$a_{p<0.05,}{ }^{b} p<0.01$ vs. Control; ${ }^{C} p<0.05,{ }^{d} p<0.01$ vs. DM-0; ${ }^{e} p<0.05$ vs. DM-12.

manufacturer's instructions. Resultant films (Eastman Kodak Co., Scientific Imaging Systems, New Haven, CT) were scanned using a flatbed scanner and images analyzed with NIH Image software. CAV-1 determination in all fractions obtained from each rat were performed at least in triplicate.

\section{Statistical analysis}

Data are expressed as mean \pm S.E.M. All analyses were performed by analysis of variance (ANOVA) followed by the Scheffé test. $\mathrm{P}<0.05$ value was considered as statistically significant.

\section{Results}

General characteristics of control and diabetic rats are summarized in Table 1. All diabetic rats demonstrated reduced weight gain, which was partly restored by insulin treatment. DM-0 demonstrated renal hypertrophy. Renal hypertrophy was completely and partially normalized by intensive insulin treatment and losartan, respectively. As expected, diabetic rats without insulin treatment displayed significant hyperglycemia. SBP was higher in diabetic rats and markedly reduced by treatment with losartan.

Western blot analysis of individual fractions obtained from control animals revealed CAV-1 expression in fractions 4-6 with the highest abundance in fraction 5 (Fig. 1). These fractions corresponded to light scattering band that typically forms after separating cellular fractions on glucose gradient. Therefore, these fractions were considered to be caveolar fractions. In control rats, CAV-1 was also detectable in fractions 8-10. These fractions, and all other fractions except caveolar fractions were considered to be non-caveolar fractions.

As it has been previously proposed by Kawabe et al. (2004) in studies conducted in VSM cells, a ratio of caveolar/non-caveolar expression of CAV-1 was then computed for each fractionated renal cortex allowing comparisons of CAV-1 subcellular distribution according to various conditions or treatments. Using this approach, severely diabetic rats demonstrated marked increases in the ratio of caveolar/non-caveolar CAV-1 as compared to control animals. These increases in CAV-1 caveolar/noncaveolar ratio were, in diabetic rats, ameliorated by intensive insulin treatment and treatment with losartan (Fig. 1).

\section{Discussion}

The unusual lipid composition of caveolae imparts to these microdomains the properties instrumental for their purification and characterization, namely a highly reduced density as compared to their phospholipid counterparts, and resistance to solubilization by mild nonionic detergents. Sucrose gradient ultracentrifugation utilizes the detergent resistance and buoyancy of these microdomains to separate them from other cellular constituents (Lisanti et al. 1994).

Our observations in normal rats, i.e. marked presence of CAV-1 in fractions 4-6, correspond to previously reported data in a variety of homogenous cell lines, such as the skeletal muscle, vascular smooth muscle and endothelial cells (Munoz et al. 1996, Ishizaka et al. 1998, Fulton et al. 2002, Sampson et al. 2004, 2007, Peng et al. 2007). This CAV-1 presence in fractions 4-6, as opposed to minimal abundance of the protein in fractions 7-8, was apparent despite the comparable total protein content in these fractions $(0.4-$ $0.8 \mu \mathrm{g} / \mu \mathrm{l})$.

With respect to the second aim of these studies, 


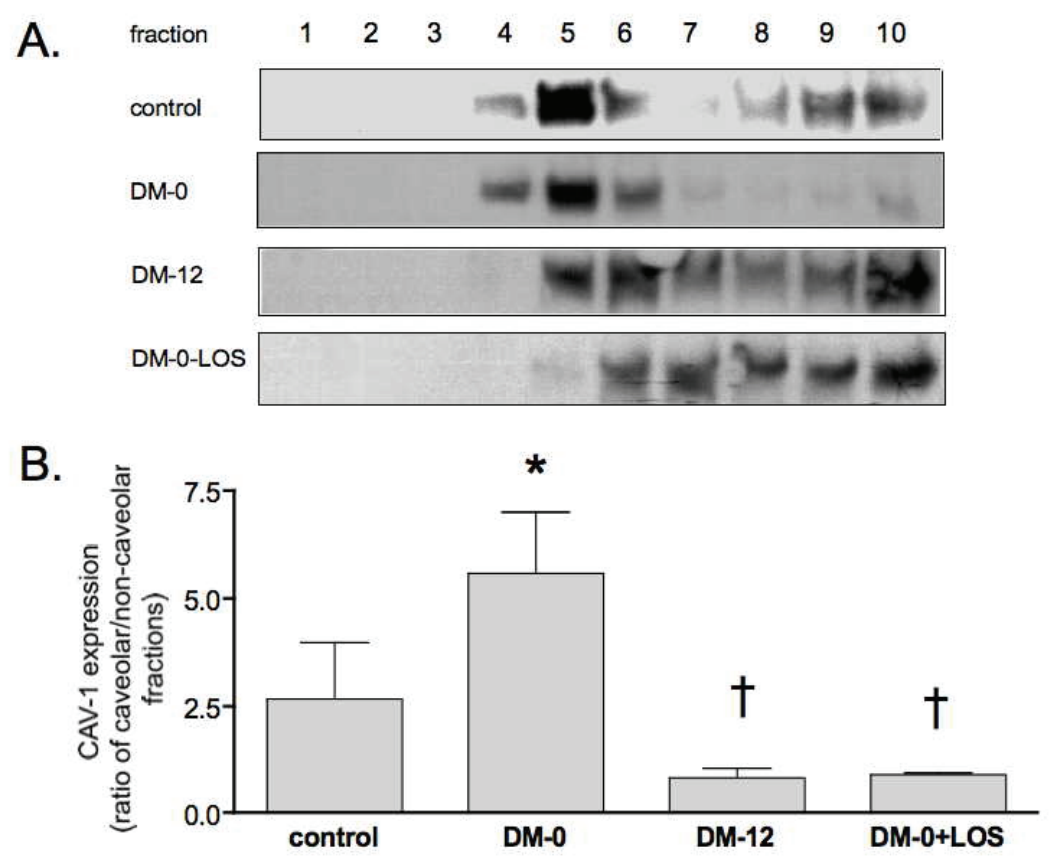

Fig. 1. Fractionation on sucrose gradient of renal cortical homogenates harvested from control and diabetic rats. Renal cortical homogenates were subjected to sucrose fractionation as described in Methods. Panel A shows representative images of CAV- 1 expression analyzed by Western blotting in subsequent (1-10) fractions in control rats, in diabetic rats without pharmacological interventions (DM-0), in diabetic rats with highdose insulin treatment to achieve tight metabolic control (DM-12), and in diabetic rats treated with angiotensin receptor blocker losartan (DM-0-LOS). Fractions 4-6 were considered to be caveolar fractions. All other fractions except caveolar fractions were considered to be non-caveolar fractions. A ratio of caveolar/non-caveolar expression of CAV-1 was then computed for each fractionated renal cortex allowing comparisons of CAV- 1 subcellular distribution according to various conditions or treatments. Panel B shows mean ratios of caveolar/non-caveolar expression of CAV-1 in each group of rats. CAV-1 determination in all fractions obtained from each rat were performed at least in triplicate. $* p<0.05$ vs. control; $+p<0.01$ vs. DM-0. we show that the fractionation on sucrose gradient can detect the changes in cellular distribution of CAV-1 induced by various disease states, e.g. in a model of Type 1 diabetes with poor metabolic control. Analysis of renal cortical fractions obtained from severely diabetic rats revealed a shift of CAV-1 from non-caveolar to caveolar fractions, as compared to non-diabetic animals.

Thus far, sucrose fractionation has not been applied in studies focusing on the pathophysiology of diabetic microvascular complications or on the role of CAV-1 in the pathogenesis of the kidney disease. Consequently, comparisons of the present data to previous studies focusing on the alterations of vascular or renal CAV-1 in diabetes is difficult. However, several previous studies have reported the data that correspond to our present observations. For example, Pascariu et al. (2004) studied CAV-1 expression in luminal aspects of endothelial cells isolated from pulmonary vasculature in the same model of diabetes as in the present study. In these membrane preparations, they found increased number of caveolae and enhanced abundance of CAV-1 protein. However, we can only speculate about the effects of diabetes caveolar/non-caveolar ratios, since the authors did not provide any information on the non-caveolar expression of CAV-1.

Finally, we report that sucrose fractionation followed by Western blotting can detect shifts in CAV-1 subcellular distribution in response to pharmacological treatments. In diabetic rats, CAV-1 caveolar/non-caveolar ratio was normalized by insulin treatment that achieved tight metabolic control. Furthermore, similar effect was observed after chronic administration of losartan.

Insulin action is closely linked to preserved caveolar integrity. There is abundant evidence indicating that organization of signaling molecules in caveolae and their interactions with caveolins are crucial for insulin receptor function (Ishikawa et al. 2005). Consequently, modulation of CAV-1 expression and subcellular localization has major impact on insulin action in a given tissue. However, the evidence exploring the mechanisms in an opposite direction, i.e. the effects of insulin on CAV-1 expression and subcellular targeting, have been far less studied. Moreover, the mechanisms of insulininduced shifts of CAV-1 in renal cells remain unknown.

In the present studies, insulin, administered in the higher dose required to achieve tight metabolic control in STZ-diabetic rats, reduced caveolar/noncaveolar ratio of CAV-1, suggesting the shift of CAV-1 into the non-caveolar locations. Supporting this notion, hyperinsulinemia due to insulin resistance in obese Zucker rats, a model of Type 2 diabetes, has been shown to be associated with lower renal CAV-1 expression ( $\mathrm{Li}$ et al. 2005). We have previously reported decreased membrane CAV-1 expression in moderately hyperglycemic STZ-diabetic rats that combine normal or even slightly elevated insulin levels with blood glucose levels 15-20 mmol/1, as compared to normal animals, and a normalization of this phenomenon in diabetic rats 
receiving intensive insulin treatment (Komers et al. 2006). These results suggest that insulin alone or in association with the components of diabetic metabolic milieu, such as hyperglycemia, act as important modulators of CAV-1 expression and subcellular targeting in the kidney.

Similar to insulin, treatment with losartan markedly reduced renal caveolar/non-caveolar ratio of CAV-1 as compared to untreated severely diabetic rats. To our knowledge, the effects of AT1R inhibition on CAV-1 subcellular distribution have not been studied. However, studies in vascular smooth muscle cells have shown that angiotensin II is involved in CAV-1 biosynthesis (Ishizaka et al. 1998). Moreover, upon agonist stimulation AT1R is redistributed to caveolae, where it interacts with CAV-1 (Ishizaka et al. 1998). Based on this evidence, it is conceivable that AT1R blockade reduces caveolar CAV-1.

Several limitations of this type of $\mathrm{CAV}-1$ determination should be pointed out. First, this method is not suitable for testing the differences in expression of CAV-1 between the different experimental conditions. To determine whether particular experimental condition or a disease leads to differences in CAV-1 expression, it is necessary to directly compare $\mathrm{CAV}-1$ protein abundance in equal amounts of total protein from corresponding fractions. Second, in organs or tissues with multiple cell types, additional immunohistochemical studies might be required to localize proteins under study and better focus interpretation of the data. We have previously shown that in the rat kidney, CAV-1 is expressed predominantly in endothelial cells, arteriolar vascular smooth muscle, glomerular epithelial cells, and in basolateral aspects of distal tubules (Komers et al. 2006). Studies by others also described abundant caveolae and CAV-1 expression in mesangial cells (Tamai et al. 2001). Therefore, these cell types must have provided most of the detected CAV-1.

In summary, present studies indicate that the method of sucrose fractionation could be applied in studies focusing on CAV-1 pathophysiology in organs and tissues that contain various cell lines, and provide information about the CAV-1 targeting under in vivo conditions, avoiding artificial conditions of the cell culture. Moreover, in addition to studies assessing the subcellular distribution of CAV-1, this method can be used for co-localization of other proteins that undergo caveolar translocation in response to a variety of physiological and pathophysiological stimuli. Present studies were not designed to test the pathophysiological consequences of differences in CAV-1 distribution. However, considering the abundant and rapidly expanding knowledge about the roles of CAV-1 in various signaling and biochemical pathways, one might speculate that the alterations in CAV-1 subcellular targeting may have major impact on a wide spectrum of signaling events and enzymatic activities in renal cells.

\section{Conflict of Interest}

There is no conflict of interest.

\section{Acknowledgements}

These studies were supported by the institutional Grant \# MZO 00023001 of the Czech Ministry of Health Care. We are grateful to J. Veselá, for her excellent technical assistance.

\section{References}

ANDERSON RGW: The caveolae membrane system. Annu Rev Biochem 67: 199-225, 1998.

COOPER ME: Pathogenesis, prevention, and treatment of diabetic nephropathy. Lancet 352: 213-219, 1998.

ENGELMAN JA, CHU C, LIN A, JO H, IKEZU T, OKAMOTO T, KOHTZ DS, LISANTI MP: Caveolin-mediated regulation of signaling along the p42/44 MAP kinase cascade in vivo. A role for the caveolin-scaffolding domain. FEBS Lett 428: 205-211, 1998.

FRANK PG, WOODMAN SE, PARK DS, LISANTI MP: Caveolin, caveolae, and endothelial cell function. Arterioscler Thromb Vasc Biol 23: 1161-1168, 2003.

FULTON D, FONTANA J, SOWA G, GRATTON JP, LIN M, LI KX, MICHELL B, KEMP BE, RODMAN D, SESSA WC: Localization of endothelial nitric-oxide synthase phosphorylated on serine 1179 and nitric oxide in Golgi and plasma membrane defines the existence of two pools of active enzyme. J Biol Chem 277: 42774284, 2002.

ISHIKAWA Y, OTSU K, OSHIKAWA J: Caveolin, different roles for insulin signal? Cell Signal 17: 1175-1182, 2005. 
ISHIZAKA N, GRIENDLING KK, LASSEGUE B, ALEXANDER RW: Angiotensin II type 1 receptor: relationship with caveolae and caveolin after initial agonist stimulation. Hypertension 32: 459-466, 1998.

KAWABE J, OKUMURA S, LEE MC, SADOSHIMA J, ISHIKAWA Y: Translocation of caveolin regulates stretchinduced ERK activity in vascular smooth muscle cells. Am J Physiol 286: H1845-H1852, 2004.

KOMERS R, SCHUTZER WE, REED JF, LINDSLEY JN, OYAMA TT, BUCK DC, MADER SL, ANDERSON S: Altered endothelial nitric oxide synthase targeting and conformation and caveolin-1 expression in the diabetic kidney. Diabetes 55: 1651-1659, 2006.

LI Z, RODRIGUEZ-ITURBE B, NI Z, SHAHKARAMI A, SEPASSI L, VAZIRI ND: Effect of hereditary obesity on renal expressions of NO synthase, caveolin-1, AKt, guanylate cyclase, and calmodulin. Kidney Int 68: 2766$2772,2005$.

LISANTI MP, SCHERER PE, VIDUGIRIENE J, TANG Z, HERMANOWSKI-VOSATKA A, TU YH, COOK RF, SARGIACOMO M: Characterization of caveolin-rich membrane domains isolated from an endothelial-rich source: implications for human disease. J Cell Biol 126: 111-126, 1994.

LOWRY OH, ROSENBROUGH WH, FARR AL, RANDALL RJ: Protein measurement with the Folin phenol reagent. J Biol Chem 193: 265-275, 1951.

MUNOZ P, MORA S, SEVILLA L, KALIMAN P, TOMAS E, GUMA A, TESTAR X, PALACIN M, ZORZANO A: Expression and insulin-regulated distribution of caveolin in skeletal muscle. Caveolin does not colocalize with GLUT4 in intracellular membranes. J Biol Chem 271: 8133-8139, 1996.

PASCARIU M, BENDAYAN M, GHITESCU L: Correlated endothelial caveolin overexpression and increased transcytosis in experimental diabetes. J Histochem Cytochem 52: 65-76, 2004.

PENG F, WU D, INGRAM AJ, ZHANG B, GAO B, KREPINSKY JC: RhoA activation in mesangial cells by mechanical strain depends on caveolae and caveolin-1 interaction. J Am Soc Nephrol 18: 189-198, 2007.

SAMPSON LJ, HAYABUCHI Y, STANDEN NB, DART C: Caveolae localize protein kinase A signaling to arterial ATP-sensitive potassium channels. Circ Res 95: 1012-1018, 2004.

SAMPSON LJ, DAVIES LM, BARRETT-JOLLEY R, STANDEN NB, DART C: Angiotensin II-activated protein kinase $\mathrm{C}$ targets caveolae to inhibit aortic ATP-sensitive potassium channels. Cardiovasc Res 76: 61-70, 2007.

SONG KS, LI S, OKAMOTO T, QUILLIAM LA, SARGIACOMO M, LISANTI MP: Co-purification and direct interaction of Ras with caveolin, an integral membrane protein of caveolae microdomains. Detergent-free purification of caveolae microdomains. J Biol Chem 271: 9690-9697, 1996.

TAMAI O, OKA N, KIKUCHI T, KODA Y, SOEJIMA M, WADA Y, FUJISAWA M, TAMAKI K, KAWACHI H, SHIMIZU F, KIMURA H, IMAIZUMI T, OKUDA S: Caveolae in mesangial cells and caveolin expression in mesangial proliferative glomerulonephritis. Kidney Int 59: 471-480, 2001. 